\title{
COMPOSITION DU GROUPE DE LECTURE CRITIQUE
}

\section{Ademe}

Michel COURILLON

Responsable Afrique subsaharienne et Océan indien - Département Pays Tiers Direction de l'Action internationale

\section{Agence universitaire de la francophonie}

Georges MALAMOUD

Directeur de la Prospective et de la Programmation de l'AUF

\section{Association française pour la FAO}

Marcel MAZOYER

Professeur honoraire de l'Institut national agronomique de Paris-Grignon Vice-président de l'Affao

\section{Conférence des grandes écoles}

Jean-Claude BUREAU

Directeur des Relations internationales Insa de Lyon

\section{Conférence des présidents d'université}

Bernard CARRIÈRE

Président de l'université Louis Pasteur

Conseil africain et malgache pour l'enseignement supérieur Jean KOUDOU Directeur des programmes au Cames

Haut Conseil de la coopération internationale Marie-Claude BABY Conseillère du Secrétaire général du Haut Conseil 


\section{Ined}

Thérèse $\mathrm{LOCOH}$

Directrice de recherche

\section{Ministère de I'Agriculture, de I'Alimentation, de la Pêche et des Affaires rurales}

Lilian PUECH

Chef du Bureau recherche et développement

Les membres du Groupe de lecture critique, désignés par le président ou le directeur général de leur établissement, ont examiné le texte du rapport puis, au cours d'une réunion qui s'est tenue à l'Académie des sciences le 18 mai 2005, ont entendu la présentation de M. François Gros, animateur du groupe de travail, et se sont exprimés.

Ils ont formulé des remarques, dont certaines ont été intégrées, avec leur accord, dans le rapport; quatre commentaires font l'objet de contributions signées : elles sont présentées ci-après. 


\title{
COMMENTAIRE DE L'ADEME
}

\author{
Michel Courillon
}

Responsable Afrique subsaharienne et Océan Indien

La place des énergies renouvelables et des énergies fossiles

De nombreux pays en développement reconnaissent un rôle important à l'énergie pour le développement économique et social; les documents stratégiques de lutte contre la pauvreté confirment ce rôle.

Les sources d'énergies renouvelables peuvent contribuer à la satisfaction de besoins sociaux de base notamment dans le secteur de l'éducation et de la santé et fournir des quantités limitées d'électricité pour les usages domestiques (éclairage, audiovisuel...).

De réels succès existent dans ce domaine, mais l'on constate aujourd'hui que, dans les pays les plus pauvres ou pour les populations les plus démunies, l'accès à ces nouveaux services s'inscrit parfois difficilement dans la durée malgré le soutien des programmes de coopération conventionnels ou la mise en place de partenariats public-privé.

Il est donc nécessaire d'élaborer en complément une approche en matière d'utilisation de l'énergie à des fins économiques dans le monde rural, ce qui permettra, grâce au recours à des sources d'énergies diversifiées, d'assurer une pérennité des programmes et ainsi de donner la possibilité aux populations rurales de sortir de la pauvreté.

Deux raisons principales conduisent à ce constat :

- aussi paradoxal que cela puisse paraître, pour réussir, les programmes d'accès à l'énergie à vocation sociale nécessitent moins de prérequis que des programmes de développement économique. Parmi ces prérequis, on peut mentionner : des services publics suffisamment structurés (santé et éducation), des compagnies locales d'électricité, des agences d'électrification aptes à développer de nouvelles approches pour le hors réseau... Lorsqu'il s'agit de développer des activités génératrices de revenus, les acteurs (entreprises, groupements de femmes. ..) doivent disposer d'un environnement favorable : existence de marchés de préférence de proximité 
quand il s'agit d'acteurs peu expérimentés, cadre réglementaire accessible non discriminant, existence d'organisations professionnelles à même d'apporter un appui pour la formation à la gestion, à la commercialisation, à la maîtrise de la qualité ;

- il existe une offre limitée en équipements adaptés, c'est-à-dire alliant fiabilité, coûts compatibles avec l'économie des filières, entretien et maintenance maîtrisable localement. On possède aujourd'hui une expérience suffisante pour quelques catégories d'équipements au premier rang desquels figurent les systèmes de pompage photovoltaïques pour l'alimentation de villages en eau potable et la petite irrigation, mais les potentialités de développement d'activés génératrices de revenus sont plus étendues, on peut citer par exemple:

- I'amélioration de la qualité et une diversification des productions artisanales,

- le développement dans les campagnes de produits artisanaux élaborés jusqu'à présent uniquement en ville grâce à la disponibilité d'électricité,

- la conservation et la transformation des productions agricoles.

Les clés pour mettre en place un programme dans le domaine de l'énergie pour le développement en milieu rural peuvent se synthétiser selon quatre conditions préalables :

- disposer d'un environnement déjà suffisamment structuré (organisation locale d'encadrement, programme de microcrédits...);

- connaître des potentialités de marchés;

- disposer des technologies adaptées;

- avoir une ressource énergétique adaptée.

Si les énergies renouvelables telles que I'hydroélectricité et l'éolien, quand la ressource existe en quantité suffisante, peuvent permettre de faire fonctionner l'ensemble des équipements de production agricole ou artisanale, ce n'est pas encore aujourd'hui le cas de l'énergie photovoltaïque car le coût d'investissement serait prohibitif pour le village. En conséquence, I'utilisation du moteur diesel est aujourd'hui nécessaire pour l'alimentation de petits ateliers artisanaux ruraux, pour actionner les plates-formes multifonctionnelles mises en place par le Pnud au Sahel... 
Afin de limiter l'impact environnemental et la dépendance vis-à-vis des énergies fossiles, la solution passe par l'utilisation de biocarburants comme l'huile de pourghère, l'huile de coton..., ou d'autres produits de substitution.

Aujourd'hui, ces carburants alternatifs ont été peu utilisés en Afrique en raison de leur coût de production comparé au coût du gasoil à la pompe, mais ce ratio évolue rapidement en leur faveur en 2005.

Des recherches sont donc à entreprendre dans les pays du Sud dans les domaines technique et socio-économique afin de mieux appréhender l'utilisation de ces ressources locales en biomasse. De plus, cette utilisation sera une source d'emplois locaux.

En complément, il est indispensable de travailler à l'amélioration des technologies énergétiques pour améliorer la rentabilité des projets des promoteurs (avec un temps de retour court inférieur à 5 ans, acceptable pour un entrepreneur). Par exemple, les techniques de séchage à fuel les moins performantes et parfois promues en Afrique peuvent consommer jusqu'à $4 \mathrm{kWh} / \mathrm{kg}$ d'eau extraite des produits à sécher. Augmenter l'efficacité énergétique, en particulier grâce à l'utilisation du gaz (avec souvent un appoint solaire), permet de diviser par deux la consommation énergétique.

Il est donc primordial de développer aujourd'hui des solutions techniques novatrices combinant les différentes énergies disponibles localement, renouvelables et fossiles si nécessaire, afin de permettre le développement du milieu rural voire périurbain des pays du Sud ainsi que la pérennité des programmes. 



\section{COMMENTAIRE DE L'AGENCE UNIVERSITAIRE DE LA FRANCOPHONIE}

\section{Michèle Gendreau-Massaloux}

\section{Recteur}

L'enseignement supérieur et la recherche en Afrique subsaharienne ont rarement été au centre des préoccupations des grandes institutions internationales et régionales, alors qu'ils constituent un facteur essentiel de développement. Cette situation pourrait évoluer favorablement et plusieurs signes le laissent à penser, aussi bien auprès de l'Union africaine et des institutions africaines, que des partenaires internationaux. Il est donc particulièrement opportun dans ce contexte de faire ressortir l'importance de la science en abordant toutes les questions qui se posent à partir de ce qui est proposé par les acteurs africains eux-mêmes, et notamment les établissements d'enseignement supérieur et de recherche. Le paysage institutionnel africain en matière de science est en cours de structuration, et il semble nécessaire d'aider ce mouvement à prendre de la vitesse.

Entre une approche unifiée qui fondrait dans un même moule tous les types d'intervention, et une simple observation de projets non coordonnés ou contradictoires, les coordinations par grand domaine se font autour d'acteurs qui associent les partenaires universitaires locaux et internationaux. Les apports multilatéraux comme ceux de l'AUF rencontrent alors, autour de projets bien identifiés, les apports bilatéraux comme ceux de la France.

Ainsi, le développement de pôles d'excellence au Sud en matière de recherche et de formation est-il un moyen de développer des équipes locales, fortes d'appuis dans des pays du Nord. Plusieurs projets internationaux sont à l'étude, par exemple celui des instituts africains des sciences et des technologies, conduit par l'Institut Nelson Mandela, avec l'appui de la Banque mondiale. La participation des établissements et des équipes d'Afrique francophone à ces projets crée des lieux viables attirant les jeunes chercheurs africains.

La coopération Sud-Sud apporte également un soutien efficace à un grand nombre de problèmes rencontrés dans les établissements africains. À ce titre, il semble profitable de développer des projets trilatéraux, associant par exemple un ou plusieurs pays africains, un pays du Maghreb et la France. De tels projets supposent un maillage du réseau des établissements concernés, et une coopération régionale et interrégionale, qui dépasse les habituels cadres nationaux. 
L'Agence universitaire de la francophonie a commencé à appuyer l'émergence de tels projets.

Beaucoup d'acteurs interviennent en Afrique dans ces domaines. Leur mode de gouvernance et leurs modalités d'intervention diffèrent, mais ils concourent tous, à leur façon, à une prise de conscience de la responsabilité des africains eux-mêmes dans l'invention de leur propre avenir. Les chercheurs et enseignants des diasporas se mobilisent pour participer à sa formation, soit à distance, soit en revenant pour de brefs séjours dans leurs pays d'origine. 
COMMENTAIRE DU CONSEIL AFRICAIN ET MALGACHE POUR L'ENSEIGNEMENT SUPÉRIEUR

\section{(CAMES)}

Mamadou Moustapha Sall

Secrétaire général

\section{Amélioration de l'efficacité de la coopération scientifique et technique}

Bien que les pistes qui sont envisagées dans le rapport pour atteindre cet objectif nous paraissent toutes d'une grande pertinence, nous insistons en particulier sur deux ou trois d'entre elles:

- le renforcement des capacités de recherche du Sud nous semble un passage obligé : deux programmes français y contribuent avec succès : Corus et AIRE Développement qui ont fait émerger ces dernières années des équipes de recherche de qualité. Accroître les moyens et les champs d'action de ces deux programmes est de nature à contribuer à la réalisation au Sud d'une expertise scientifique ;

- une meilleure intégration des jeunes chercheurs du Sud aux plates-formes françaises de recherche opérant au Sud (IRD et divers autres programmes de recherche scientifique). C'est aussi un moyen de prendre en charge la formation à la recherche;

- l'aide au retour : son efficacité nous paraît conditionnée à l'existence au Sud de plates-formes de recherche performantes. Sans possibilité d'une réelle valorisation au plan scientifique mais aussi économique et social des compétences acquises par les chercheurs du Sud, la fuite des cerveaux sera difficile à juguler.

Les programmes mis en œuvre dans certains pays du Sud (Sénégal, Mali, etc.) et visant à créer des liens institutionnels avec leur diaspora scientifique aux fins d'une exploitation de leur expertise méritent d'être multipliés et soutenus. 


\section{Renforcement de la formation universitaire}

Nous partageons le diagnostic porté sur la situation des universités d'Afrique subsaharienne confrontées à des difficultés de tout genre et que les réformes et refondations successives $n^{\prime}$ ont pas permis de réduire. Dans beaucoup d'entre elles, la situation de la recherche et de l'enseignement est critique.

Les interventions en cours par le biais des conventions interuniversitaires et des thèses en cotutelles sont sans doute bénéfiques. Mais pour plus d'efficacité, ne faudrait-il pas agir surtout dans le cadre des évolutions en cours? Aujourd'hui, dans l'espace Cames (17 pays francophones d'Afrique subsaharienne), des engagements politiques forts se sont exprimés en faveur des réformes académiques devant mener au passage au système $L M D$, à la suite des séminaires de Dakar (décembre 2003) et Yaoundé (mars 2005) ayant réuni sous l'égide du Cames et de l'AUF les recteurs des universités d'Afrique subsaharienne et de l'Océan indien (Madagascar). Tous perçoivent ces réformes comme nécessaires et comme une opportunité de donner à leurs universités un nouvel élan en conférant à leurs offres de formation de nouvelles pertinences économiques et sociales.

Les accompagner dans cette phase décisive serait de nature à contribuer à renforcer la formation universitaire au sud.

\section{Sur l'avis et les recommandations}

1. Le Cames, organisme d'intégration des systèmes éducatifs africains ne peut qu'appuyer les recommandations 4 et 5 du rapport, recommandations relatives à la création d'un Conseil de recherche africain d'une part, au développement des moyens nécessaires au maintien de liens permanents entre les communautés scientifiques anglophones et francophones d'autre part.

2. Le Cames appuie très fortement la suggestion que « sous l'égide de la CPU, en liaison avec les services des ministères et des représentants des universités africaines (par exemple du Cames), une série de travaux soient engagés pour étudier les propositions de ce rapport et examiner les moyens de les mettre en œuvre ».

Le Cames exprime sa disponibilité, celle de sa Conférence des recteurs et de sa Conférence des responsables nationaux des systèmes de recherche à participer à cette réflexion. 
3. Le Cames se félicite de ce que l'Académie des sciences recommande de conforter son rôle.

Le rôle du Cames peut être conforté à travers un renforcement de ses capacités institutionnelles permettant une meilleure gestion de ses programmes d'harmonisation des systèmes d'éducation et de recherche (concours d'agrégation, reconnaissance et équivalence des diplômes, gestion des carrières des enseignants et chercheurs, etc.), son accompagnement dans ses nouvelles missions de pilotage des réformes académiques vers le système $L M D$, en le dotant de capacités en évaluation, conformément à une décision de son conseil des ministres d'avril 2005 qui a décidé de la création d'une commission d'évaluation des établissements et des structures de recherche. 



\title{
| COMMENTAIRE DU HAUT CONSEIL DE LA COOPÉRATION INTERNATIONALE
}

\author{
Marie-Claude Baby
}

Conseillère

Le Haut Conseil de la coopération internationale a pris connaissance avec beaucoup d'intérêt du rapport "Sciences et pays en développement " réalisé par l'Académie des sciences. II s'agit d'un rapport remarquable et indispensable car il remet sur le devant de la scène l'importance de la recherche pour le développement durable des pays du Sud, héritière de la recherche pour le développement et de la recherche en coopération, qui ne figure pas aujourd'hui parmi les priorités de la recherche française.

En décembre 2004, le Haut Conseil avait remis un avis au Premier ministre sur ce thème, dont les conclusions sont reprises dans le rapport. La démarche de l'Académie des sciences prolonge et enrichit cette réflexion sur la question des contenus de la coopération scientifique avec les pays en développement.

Dans son ensemble, ce rapport constitue une contribution très utile pour notre coopération scientifique avec l'Afrique, dont la France a fait la priorité de son aide publique au développement.

La recherche pour le développement durable des pays du Sud a fait récemment l'objet $d$ 'une réflexion renouvelée entre le ministère de la Recherche et le ministère des Affaires étrangères, reprise dans les conclusions du Cicid (Comité interministériel pour la coopération internationale et le développement) du 18 mai 2005. Elle n'est pas encore devenue pour autant une priorité de la recherche française. C'est pourquoi ce rapport est très important.

Dans sa première partie, le rapport établit un diagnostic sur l'état des sciences en Afrique, et explique les conséquences de la désinstitutionalisation de la science sur la formation des élites et la production scientifique. La science n'a pas disparu en Afrique, mais les conditions de production ont changé, I'activité est moins tournée vers la pédagogie et la publication. Ce paysage a des conséquences sur les formes de coopération à promouvoir pour revivifier des institutions universitaires africaines. Il faut pour cela commencer par identifier et localiser les compétences existantes. Le Haut Conseil partage l'ensemble de ces analyses. 
La deuxième partie examine dans plusieurs domaines scientifiques l'effort de coopération scientifique de la France. C'était un exercice particulièrement difficile tant l'information est éclatée entre les activités des organismes et celles des universités. Les contributions sont particulièrement riches, même si tous les domaines scientifiques ne sont pas abordés.

Cette partie, essentielle, gagnerait en lisibilité et efficacité pour les destinataires du rapport si elle définissait des pistes de recherche scientifique par grands domaines susceptibles d'orienter des coopérations et/ou de faire l'objet d'appels d'offres spécifiques sur des thèmes réellement prioritaires et décisifs pour les pays en développement, en particulier dans le cadre de l'Agence nationale de la recherche. Un récapitulatif serait très utile à cet effet.

La réflexion consacrée aux conditions d'un renouveau de la science en Afrique spécifie bien les problèmes et les enjeux. La recherche à destination du Sud est devenue partie prenante de la plupart des recherches sur les biens publics mondiaux. Cette analyse devrait aider à convaincre les sceptiques du bien-fondé scientifique et politique de cette recherche.

Le Haut Conseil est pleinement en accord avec l'ensemble des recommandations de la dernière partie. Certaines de ces recommandations lui paraissent cependant devoir être soulignées compte tenu des enjeux actuels de la réforme de notre politique de recherche.

Il s'agit en particulier de la proposition d'identifier la recherche pour le développement dans la Loi d'orientation et de programmation de la recherche ainsi que dans les objectifs et la mise en œuvre de programmes de l'Agence nationale de la recherche (ANR).

En effet, le projet de loi, qui a circulé en janvier/février 2005, n'en faisait aucune mention. Par ailleurs, le collectif «Sauvons la recherche » est peu sensibilisé au sujet face aux autres enjeux majeurs de la loi pour la communauté scientifique. Enfin, les appels d'offres lancés par l'ANR ne font pas mention du partenariat avec les pays du Sud, ni de problématiques scientifiques intéressant explicitement le Sud, à l'exception de l'appel à projets " programme fédérateur agriculture et développement durable » qui précise être " aussi ouvert aux problématiques scientifiques de régions françaises et de pays étrangers et en particulier celles des pays du Sud». Cette ouverture au Sud reste cependant modeste.

Dans le cadre de la Lolf, et plus précisément dans le document budgétaire de la Mission interministérielle recherche et enseignement supérieur, qui se substitue au jaune " état de la recherche et du développement technologique ", il convient 
également de veiller à inscrire la contribution de la coopération scientifique au développement.

Le rapport propose la mise en place d'une structure de pilotage national de caractère interministériel. Celle-ci est indispensable à la conduite d'une véritable politique de recherche pour le développement. Il convient de s'assurer de la mise en œuvre des conclusions du Cicid sur ce point.

Plusieurs recommandations portent sur l'édification d'un espace africain de la recherche et sur le renforcement de la coopération interuniversitaire. Elles constituent un guide pour nos actions de coopération qui devrait utilement inspirer le ministère chargé de la Recherche et le ministère chargé des Affaires étrangères.

Sur ce thème, afin de contribuer très concrètement à l'émergence de cet espace africain de la recherche, il serait utile de pouvoir disposer d'un état des lieux des compétences scientifiques en Afrique dans les grands domaines étudiés. Cette cartographie scientifique permettrait de réfléchir avec les partenaires africains, notamment dans le cadre du Nepad', aux regroupements qu'il conviendrait de proposer pour constituer des pôles de recherche.

Enfin, le Haut Conseil note avec satisfaction que l'Académie des sciences propose la création d'une structure interacadémique chargée d'une mission de réflexion et de proposition propre à la réalisation de ces recommandations.

Le Haut Conseil remercie l'Académie des sciences de l'avoir associé à la réflexion critique sur ce sujet de la recherche pour le développement qui lui tient particulièrement à cœur.

\footnotetext{
${ }^{1}$ Nepad: Nouveau partenariat pour le développement de l'Afrique, dans lequel s'inscrivent les relations entre les pays du $G 8$ et l'Afrique.
} 
